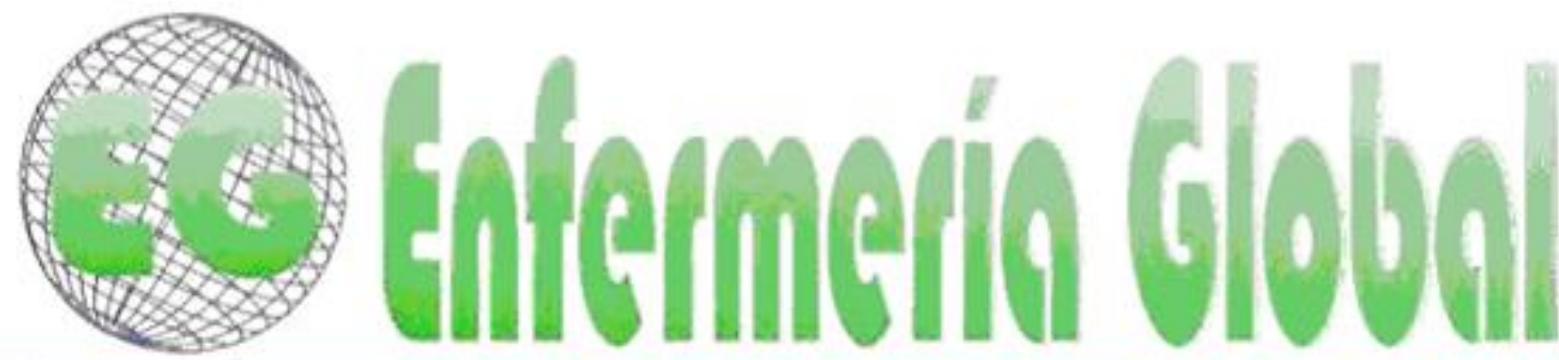

ISSN 1695-6141

Revista electrónica trimestral de Enfermeria

$\mathrm{N}^{\circ} 34$

Abril 2014

www.um.es/egloball

REVISIONES

\title{
Acciones $y / 0$ intervenciones de enfermería para la prevención de infecciones hospitalarias en pacientes gravemente enfermos: una revisión integrativa
}

Ações e/ou intervenções de enfermagem para prevenção de infecções hospitalares em pacientes gravemente enfermos: uma revisão integrativa

Shares and / or nursing interventions for prevention of nosocomial infections in critically ill patients: an integrative review

\section{${ }^{*}$ Calil, Keila ${ }^{* *}$ Cavalcanti Valente, Geilsa Soraia ${ }^{* * *}$ Silvino, Zenith Rosa}

*Enfermera. ***Doctora en Enfermería. Profesora Adjunta. E-mail: geilsavalente@yahoo.com.br ***Doctora en Enfermería. Profesora Titular. Escuela de Enfermería Aurora de Afonso Costa. Universidad Federal Fluminense/ UFF. Brasil.

Palabras clave: infección hospitalaria; unidad de cuidados intensivos; enfermería

Palavras-chave: infecção hospitalar; unidade de terapia intensiva; enfermagem

Keywords: infection; intensive care unit, nursing.

\section{RESUMEN}

Objetivo: Identificar en la literatura publicada desde 1997 las acciones o intervenciones de enfermería para la prevención de las infecciones nosocomiales en pacientes adultos en estado crítico.

Método: Revisión integradora de las fuentes de información por parte de la BVS (Biblioteca Virtual en Salud), incluyendo IBECS LILACS, Cochrane Library, MEDLINE y PubMed y SciELO. Se utilizaron las siguientes palabras clave: Infección hospitalaria, unidad de cuidados intensivos y enfermería, en un recorte temporal de 1997 a 2011.

Resultados: Se analizaron 29 artículos, 28 estaban disponibles en línea completa y la revista está disponible en la biblioteca. Para analizar los datos obtenidos se utilizó la lectura interpretativa y el análisis temático emergiendo las siguientes categorías: higiene de las manos, intervenciones educativas, introducción de las Nuevas Tecnologías en Salud, Higiene Bucal, aspiración de contenido gástrico, decúbito y el cambio de elevación de la cabecera de la cama.

Conclusión: Es posible concluir que las acciones o intervenciones de enfermería para la prevención de las infecciones nosocomiales que con mayor frecuencia aparecen en la literatura están relacionadas con las topografías torrente sanguíneo y tracto respiratorio además de lavado de manos y ejercen influencia en la reducción de la incidencia de infecciones nosocomiales. Existen pocos estudios en Brasil, siendo necesarios incentivar las nuevas investigaciones en esta área. 


\section{RESUMO}

Objetivo: Identificar na literatura existente publicada a partir do ano de 1997, as ações e/ou intervenções de enfermagem para prevenção de infecções hospitalares em pacientes adultos criticamente enfermos.

Método: Revisão integrativa através das fontes de informação BVS (Biblioteca Virtual em Saúde) incluindo LILACS, IBECS, Biblioteca Cochrane, SciELO e MEDLINE e PubMed. Foram utilizados os seguintes descritores: infecção hospitalar, unidade de terapia intensiva e enfermagem utilizando um recorte temporal do ano de 1997 a 2011.

Resultados: Foram analisados 29 artigos sendo que 28 estavam disponíveis na íntegra online e um de revista disponível na biblioteca. Para analisar os dados encontrados utilizou-se a leitura iterpretativa e a análise temática emergindo as seguintes categorias: Higienização das Mãos, Intervenções Educacionais, Introdução de Novas Tecnologias em Saúde, Higiêne Oral, Aspiração do Conteúdo Gástrico, Mudança de Decúbito e Elevação da Cabeceira do Leito.

Conclusão: É possível concluir que as ações e/ou intervenções de enfermagem para prevenção de infecções hospitalares que mais aparecem na literatura estão relacionadas as topografias corrente sanguínea e trato respiratório além da lavagem das mãos e, exercem influência na redução da incidência de infecções hospitalares. Existem poucos estudos realizados no Brasil, sendo necessário incentivo à novas pesquisas nesta área.

\section{ABSTRACT}

Objective: To identify the literature published from 1997, the actions and / or nursing interventions for prevention of nosocomial infections in critically ill adult patients.

Method: an integrative review of information sources by VHL (Virtual Health Library) including LILACS IBECS, Cochrane Library, MEDLINE and PubMed and SciELO. We used the following keywords: hospital infection, intensive care unit and / nursing using a time frame of 1997 to 2011.

Results: We analyzed 29 articles and 28 were available in full online and magazine available in the library. To analyze the data obtained the interpretative reading and the thematic analysis was used, arising the following categories: Hand Hygiene, Educational Interventions, Introduction of New Technologies in Health, Oral Hygiene, Aspiration of Gastric Contents, Decubitus and Elevation Change of the headboard of the bed.

Conclusion: It is possible to conclude that the actions and / or nursing interventions for prevention of nosocomial infections that most often appear in the literature are related topographies bloodstream and respiratory tract than washing of hands and influence in reducing the incidence of nosocomial infections. There are few studies in Brazil, being necessary incentives for further research in this area.

\section{INTRODUCCIÓN}

La infección hospitalaria representa uno de los principales problemas de la asistencia a la salud con el consiguiente impacto en la morbidad y mortalidad de los pacientes ingresados en los hospitales, principalmente en aquellos gravemente enfermos.

Los episodios de infección hospitalaria se concentran en Unidades de Cuidados Intensivos (UCI) lo que torna a estos servicios en prioritarios para las acciones de prevención y control de infecciones. Este hecho está relacionado con la mayor gravedad y permanencia de los pacientes ingresados además de la realización de múltiples procedimientos invasivos ${ }^{(1)}$.

La Agencia Nacional de Vigilancia Sanitaria (ANVISA) establece Indicadores Nacionales de Infecciones Relacionadas con la Salud (IRAS) a través del Manual: Indicadores Nacionales de IRAS. Incluye Infección Primaria de Corriente Sanguínea 
(IPCS) en pacientes en uso de Catéter Venoso Central (CVC) así como vigilancia y notificación obligatoria en el ámbito nacional para todos los establecimientos de salud, públicos o privados, con unidades de cuidado intensivo neonatal, pediátrica y adulto que totalicen o aisladamente posean 10 (diez) o más camas. Recomienda también vigilancia de otros indicadores como: Pneumonía Asociada a Ventilación Mecánica (PAVM) e Infección del Tracto Urinario (ITU) asociado a Catéter Vesical de Demora (CVD). Estos indicadores son llamados indicadores de resultado y permiten la realización de un diagnóstico situacional de infección hospitalaria ${ }^{(2)}$.

Pensando en mejorar los resultados y desarrollar planes de acción para la mejora de la práctica, la ANVISA establece la importancia de la evaluación de proceso por medio de indicadores y establece qué indicadores de proceso pueden ser comprendidos, así como la evaluación de intervenciones y/o acciones que lleven a un buen 0 mal resultado ${ }^{(3-4)}$.

Para la descripción de indicadores de proceso, debe considerarse la importancia del problema a ser evaluado, el impacto de determinada medida bajo la ocurrencia estudiada, el potencial para la implementación de medidas de prevención y control, las exigencias de la legislación nacional y referencias científicas ${ }^{(3-4)}$.

Cuando se piensa en acciones y/o intervenciones para la prevención de infecciones hospitalarias en UCI enseguida hay una relación con el cuidado de enfermería ya que en su ejecución hay una preocupación por la recuperación del paciente y una asistencia libre de daños ${ }^{(5)}$. El cuidado a la cabecera del enfermo y los modos de preservar la salud son objeto de constantes reflexiones para el desarrollo de concepciones teóricas iniciales en la enfermería que todavía hoy, por ser revolucionarias, inspiran la enfermería contemporánea.

Ante la importancia de esta temática esta revisión integrativa forma parte de una profundización inicial que dará soporte al proyecto de investigación intitulado provisionalmente como: Implicaciones de las acciones e intervenciones de enfermería en la ocurrencia de infección hospitalaria: análisis del servicio de una unidad de cuidados intensivos de un hospital público, que está siendo desarrollado junto al Programa de Máster Profesional en Enfermería Asistencial de la Escuela de Enfermería Aurora de Afonso Costa de la Universidad Federal Fluminense - UFF/ Niterói/RJ, que busca analizar las acciones y/o intervenciones de enfermería relacionadas con la prevención de infecciones hospitalarias en adultos enfermos en estado crítico.

El presente estudio tiene como objetivo identificar en la literatura existente, publicada a partir del año 1997, las acciones y/o intervenciones de enfermería para la prevención de infecciones hospitalarias en pacientes adultos en estado crítico.

\section{METODOLOGÍA}

Se trata de una revisión integrativa de literatura que es definida como un amplio abordaje metodológico por permitir la evaluación de estudios experimentales y no experimentales para una comprensión completa del fenómeno analizado generando un panorama consistente y comprensible de problemas de salud relevantes para la enfermería ${ }^{(6)}$. 
Para la búsqueda de los artículos se utilizó la siguiente pregunta norteadora: ¿Cuáles son las acciones y/o intervenciones de la enfermería para la prevención de las infecciones hospitalarias en adultos enfermos en estado crítico?

Se realizó una investigación en fuentes de información electrónica: Biblioteca Virtual em Saúde - BVS y PUBMED con la utilización de los siguientes descriptores respectivamente: Infección Hospitalaria, Unidad de Cuidado Intensivo y /enfermería; Hospital Infection, Critical Care y Nursing utilizando "and" para ligación de las palabras.

En la BVS, como criterios de inclusión, se utilizó: artículos originales, revisión de literatura en los idiomas portugués, inglés y español, con recorte temporal de 1997 a 2011. Se tuvo en consideración para este recorte la Ley Federal $n^{\circ} 9.431$ que dispone sobre la obligatoriedad del mantenimiento del programa de control de infecciones hospitalarias por los hospitales del País que entró en vigor en 1997. Como criterios de exclusión se utilizó: estudios con animales, estudios con recién nacidos y niños, estudios que relacionaban infección con carga de trabajo, artículos que abordaban precauciones de aislamiento, artículos repetidos.

En la PubMed, se utilizó como criterios de inclusión: artículos originales, revisión de literatura, artículos con texto completo disponibles online e investigación con humanos en los idiomas portugués, inglés y español, con recorte temporal de 1997 a 2011. Como criterios de exclusión se utilizó: estudios con recién nacidos y niños, estudios que relacionaban infección con carga de trabajo, artículos que abordaban precauciones de aislamiento, artículos repetidos.

Los artículos que abordan precauciones de aislamiento fueron excluídos por considerar que esta temática debe ser trabajada separadamente ya que presenta aspectos específicos para su análisis que este trabajo no contempla.

Para la colecta de las informaciones de cada estudio seleccionado se utilizó un instrumento en forma de planilla ${ }^{(7)}$ disponibilizado por la profesora responsable de la disciplina obligatoria Tópicos de Actualización Programada cursada en el primer semestre del Máster Profesional en Enfermería Asistencial de la Escuela de Enfermería de la UFF/Niterói/RJ. Este instrumento incluía como tópicos para rellenado de los autores, el objetivo del estudio, el tamaño de la muestra, las características y el escenario estudiado, el diseño y los instrumentos de la investigación, los principales hallazgos y las conclusiones.

De ello se desprende el análisis crítico de los estudios, la discusión de los resultados y la presentación de los datos.

\section{RESULTADOS Y DISCUSIÓN}

Utilizando la combinación de los tres descriptores se encontraron en la BVS 111 artículos y en la PubMed 833. Seguidamente se aplicaron los criterios de inclusión propuestos lo que mostró un total de 50 artículos en la BVS y 48 en la PubMed. Tras la aplicación de los criterios y exclusión se obtuvieron 10 artículos en la BVS y 19 en la PubMed. De los artículos encontrados en la BVS, 8 estaban disponibles en la base de datos MEDLINE, 1 en IBECS y 1 en SciElo. 
Vale resaltar que de los 10 artículos encontrados en la BVS, ninguno estaba disponible con texto completo por lo que fue necesario buscar en Google Académico donde se encontraron 4, en la biblioteca de la UFF estaban disponibles 5 online y 1 fue conseguido a través de la revista disponible también en la biblioteca de la Escuela de Enfermería de la UFF.

En relación a la cronología de los artículos, 2 fueron publicados en el año 2002, 3 en 2003, 2 en 2004, 1 en 2005, 2 en 2006, 6 en 2007, 3 en 2008, 2 en 2009, 4 en 2010 y 3 en 2011.

En relación a las fuentes de datos, se constató que la mayor parte se concentra en los Estados Unidos de América - USA 23, en España 2, en Italia 1, en Polonia 1, en Inglaterra 1 y 1 en Brasil.

Sobre los autores 55\% (16) estudios fueron realizados por enfermeros, $14 \%$ (4) por médicos y $31 \%$ (9) por 2 o más profesionales del área de salud.

Al analizar el diseño de las investigaciones fue posible verificar que $45 \%$ (13) de los estudios fueron observacionales, 30\% (9) estudios descriptivos, 14\% (4) revisión integrativa, 7\% (2) ensayo clínico y 3\% (1) revisión sistemática.

Como escenario se obtuvo que $42 \%$ (12) de las investigaciones realizadas fueron en hospital universitario, 34\% (10) en hospitales no universitarios, 14\% (4) investigación realizada en banco de informaciones online y 10\% (3) realizadas durante congresos y seminarios. Vale resaltar que cuando ocurridas en hospitales todas fueron realizadas en unidades de pacientes críticos.

Para analizar los datos encontrados, se utilizó la lecura interpretativa y el análisis temático. Fue posible así categorizar los datos de acuerdo con las acciones y/o intervenciones para prevención de infecciones hospitalarias que presentaban los estudios: higiene de las manos, medidas educativas con enfoque para prevención de Infección de Corriente Sanguínea (ICS) asociada al Catéter Venoso Central (CVC) y prevención de Pneumonía Asociada a Ventilación Mecánica (PAVM), administración de medicamentos, higiene oral, elevación de la cabecera del lecho, cambio de decúbito, aspiración del contenido gástrico.

\section{Higiene de las Manos}

Se describieron dos artículos que abordan la higiene de las manos realizada con agua y jabón y la posibilidad del uso de fricción con alcohol como forma de mejorar la adherencia a la técnica.

\begin{tabular}{|c|c|c|c|c|}
\hline AUTORES & AÑO & $\begin{array}{l}\text { BASE DE } \\
\text { FUENTE DE }\end{array}$ & $\begin{array}{l}\text { DATOS/ } \\
\text { DATOS }\end{array}$ & TÍTULO \\
\hline $\begin{array}{l}\text { Qushmaq IA } \\
\text { Heels-Ansdell } \\
\text { D } \\
\text { Cook DJ } \\
\text { Loeb MB } \\
\text { Meade MO }\end{array}$ & 2008 & $\begin{array}{l}\text { MEDLINE/ } \\
\text { Med Wewn }\end{array}$ & Pol Arch & $\begin{array}{l}\text { Hand hygiene in intensive } \\
\text { care unit: prospective } \\
\text { observations if clinical } \\
\text { practice }\end{array}$ \\
\hline Hugonnet S & 2002 & MEDLINE/ A & Arch Intern & Alcohol-based \\
\hline
\end{tabular}




\begin{tabular}{l|l|l|l}
\hline $\begin{array}{l}\text { Perneger TV } \\
\text { Pittet D (8) }\end{array}$ & & Med & $\begin{array}{l}\text { improves compliance with } \\
\text { hand in intensive care units }\end{array}$ \\
\hline
\end{tabular}

Cuadro I - Distribución de las bibliografías de la categoría temática "Higiene de las Manos".

Estudio desarrollado en Polonia ${ }^{(8)}$ en el que se observó a 115 profesionales de salud durante cada atención al paciente. Se registró el lavado de las manos con agua y jabón, la fricción con alcohol y el uso de guantes. El objetivo fue medir la adherencia a la recomendación de higiene de las manos entre los profesionales de UCl. La tasa de adhesión a las recomendaciones fue de $20 \%$. Ante lo observado fue posible concluir que son necesarios abordajes multidisciplinares destinados a mejorar la adhesión a la higiene de las manos.

Estudio desarrollado en los Estados Unidos ${ }^{(9)}$ en el que se observó la higiene de las manos de los profesionales con agua y jabón o la fricción con alcohol durante el cuidado a los pacientes. En seguida, se realizó una campaña para presentar los datos observados, con uso de carteles y distribución de frascos de alcohol individuales. EI objetivo fue evaluar el efecto de una intervención para incentivar la higiene de las manos e investigar los factores de riesgo para la no adhesión en la $\mathrm{UCl}$. La conformidad de higiene de las manos aumentó de $38,4 \%$ a $54,5 \%$ con el estudio. El lavado de las manos con agua y jabón permaneció en torno al 30\% mientras que la fricción con alcohol aumentó del 5,4\% a 21,7\%. La higiene de las manos con agua y jabón disminuyó una media de 4,7\% para cada 10 oportunidades por hora de asistencia al paciente siendo que esto no ocurrió con la fricción de las manos con alcohol. La intervención indujo un aumento significativo y sustentado de la conformidad de la higiene de las manos. En UCl la fricción con alcohol es más rápida y puede sustituir al lavado estandar con agua y jabón.

La higiene de las manos es la principal acción para prevención de infecciones hospitalarias y la preocupación actual está relacionada con su adhesión por los profesionales que prestan cuidados directos a los pacientes en riesgo de adquirir estas infecciones. Las estrategias son descritas para posibilitar el simplificar la práctica y la ejecución correcta de esta acción.

\section{Intervenciones Educativas}

Se presentaron trece artículos que abordan intervenciones educativas como estrategia para interferir en la ocurrencia de las infecciones hospitalarias.

\begin{tabular}{|c|c|c|c|}
\hline AUTORES & AÑO & $\begin{array}{l}\text { BASE DE DATOS/ } \\
\text { FUENTE DE DATOS }\end{array}$ & TÍTULO \\
\hline $\begin{array}{l}\text { Vandijck DM } \\
\text { Labeau SO } \\
\text { Vogelaers DP } \\
\text { Blot SI }^{(10)}\end{array}$ & 2010 & $\begin{array}{l}\text { MEDLINE/ Nursing in } \\
\text { Critical Care }\end{array}$ & $\begin{array}{l}\text { Prevention of nosocomial } \\
\text { infections in intensive care } \\
\text { patients }\end{array}$ \\
\hline $\begin{array}{l}\text { Warren DK } \\
\text { Zack JE } \\
\text { Cox MJ } \\
\text { Cohen MM } \\
\text { Fraser VJ } \\
\end{array}$ & 2003 & $\begin{array}{l}\text { MEDLINE/ Crit Care } \\
\text { Med }\end{array}$ & $\begin{array}{l}\text { An educational intervention } \\
\text { to prevent catheter- } \\
\text { associated bloodstream } \\
\text { infections in a nonteaching, } \\
\text { community medical center }\end{array}$ \\
\hline
\end{tabular}




\begin{tabular}{|c|c|c|c|}
\hline Zack JE $^{(12)}$ & 2008 & MEDLINE/ AJIC & $\begin{array}{l}\text { Zeroing in on zero tolerance } \\
\text { for central line-associated } \\
\text { bacteremia }\end{array}$ \\
\hline $\begin{array}{l}\text { Coopersmith CM } \\
\text { Zack JE } \\
\text { Ward MR } \\
\text { Sona CS } \\
\text { Scallon ME } \\
\text { Everett SJ } \\
{ }^{(13)}\end{array}$ & 2004 & MEDLINE/ Arch Surg & $\begin{array}{l}\text { The impact if bedside } \\
\text { behavior on catheter-related } \\
\text { bacteremia in the intensive } \\
\text { care unit }\end{array}$ \\
\hline $\begin{array}{l}\text { Warren DK } \\
\text { Zack JE } \\
\text { Mayfield JL } \\
\text { Chen A } \\
\text { Prantice D } \\
\text { Fraser JV } \\
\text { Kollef } \mathrm{MH}^{(14)}\end{array}$ & 2004 & MEDLINE/ Chest & $\begin{array}{l}\text { The effect of an education } \\
\text { program on the incidence of } \\
\text { central venous catheter- } \\
\text { associated bloodstream } \\
\text { infection in a medical ICU }\end{array}$ \\
\hline $\begin{array}{l}\text { Koutzavekiaris I } \\
\text { Vouloumanou } \\
\text { EK } \\
\text { Gourni M } \\
\text { Rafailidis PI } \\
\text { Michalopoulos A } \\
\text { Falagas ME }{ }^{(15)}\end{array}$ & 2011 & $\begin{array}{l}\text { MEDLINE/ Am J } \\
\text { Infect Control }\end{array}$ & $\begin{array}{l}\text { Knowledge and practices } \\
\text { regarding prevention of } \\
\text { infections associated with } \\
\text { central venous catheters: a } \\
\text { survey of intensive care unit } \\
\text { medical and nursing staff }\end{array}$ \\
\hline $\begin{array}{l}\text { Castilho LD } \\
\text { Medeiros MM } \\
\text { Carrasco HVCJ } \\
\text { Marvulo MML }{ }^{(16)}\end{array}$ & 2009 & SciElo/ Nursing & $\begin{array}{l}\text { Infecções de corrente } \\
\text { sanguínea relacionadas ao } \\
\text { cateter venoso central em } \\
\text { terapia intensiva: ensaio } \\
\text { clínico randomizado aberto }\end{array}$ \\
\hline $\begin{array}{l}\text { Llauradó M } \\
\text { Labeau S } \\
\text { Vandijck D } \\
\text { Rello J } \\
\text { Rosa A } \\
\text { Riera A }{ }^{(17)} \text { et al }\end{array}$ & 2011 & $\begin{array}{l}\text { MEDLINE/ Med } \\
\text { Intensiva }\end{array}$ & $\begin{array}{l}\text { Southern european intensive } \\
\text { care nurses' knowledge of } \\
\text { evidence-based gudelines } \\
\text { for preventing ventilator- } \\
\text { associated pneumonia }\end{array}$ \\
\hline $\begin{array}{l}\text { Zaydfudim V } \\
\text { Dossett LA } \\
\text { Starmer JM } \\
\text { Arbogast PG } \\
\text { Feurer ID } \\
\text { Ray WA }{ }^{(18)} \text { et al }\end{array}$ & 2009 & MEDLINE/ Arch Surg & $\begin{array}{l}\text { Implementation of a real- } \\
\text { time compliance dashboard } \\
\text { to help reduce SICU } \\
\text { ventilator-associated } \\
\text { pneumonia with the } \\
\text { ventilator bundle }\end{array}$ \\
\hline $\begin{array}{l}\text { Cason CL } \\
\text { Tyner T } \\
\text { Saunders S } \\
\text { Broome L }{ }^{(19)}\end{array}$ & 2007 & $\begin{array}{l}\text { MEDLINE/ Am J Crit } \\
\text { Care }\end{array}$ & $\begin{array}{l}\text { Nurses' implementation of } \\
\text { guidelines for ventilator- } \\
\text { associated pneumonia from } \\
\text { the Centers for Disease } \\
\text { Control and Prevention }\end{array}$ \\
\hline $\begin{array}{l}\text { Biancofire G } \\
\text { Barsotti E } \\
\text { Catalani V } \\
\text { Landi A } \\
\text { Bind L } \\
\text { Urbani L }{ }^{(20)} \mathrm{e}\end{array}$ & 2007 & $\begin{array}{ll}\text { MEDLINE/ Minerva } \\
\text { Anestesiol }\end{array}$ & $\begin{array}{l}\text { Nurses' } \\
\text { application of ed evidence- } \\
\text { based guidelines for } \\
\text { preventing a ventilator- } \\
\text { associated pneumonia }\end{array}$ \\
\hline
\end{tabular}




\begin{tabular}{|c|c|c|c|}
\hline $\begin{array}{l}\text { Labeau S } \\
\text { Vandijck DM } \\
\text { Claes B } \\
\text { Van Aken P } \\
\text { Blot SI }{ }^{(21)}\end{array}$ & 2007 & $\begin{array}{l}\text { MEDLINE/ Am J Crit } \\
\text { Care }\end{array}$ & $\begin{array}{l}\text { Critical care } \\
\text { knowledge of evidence- } \\
\text { based guidelines for } \\
\text { preventing } \\
\text { associated pneumonia: an } \\
\text { evaluation questionnaire }\end{array}$ \\
\hline $\begin{array}{l}\text { Tolentino- } \\
\text { DelosReyes AF } \\
\text { Ruppert SD } \\
\text { Shiao SY }{ }^{(22)}\end{array}$ & 2007 & $\begin{array}{l}\text { MEDLINE; Am J Crit } \\
\text { Care }\end{array}$ & $\begin{array}{l}\text { Evidence-based practice use } \\
\text { of the ventilator bundle to } \\
\text { prevent ventilator-associated } \\
\text { pneumonia }\end{array}$ \\
\hline
\end{tabular}

Cuadro II - Distribución de las bibliografías de la categoría temática "Intervenciones Educativas".

En estudio desarrollado en Bélgica ${ }^{(10)}$ se aplicó un test de conocimento para los enfermeros de $\mathrm{UCl}$ respetando las recomendaciones basadas en la evidencia para prevención de infecciones nosocomiales con foco especial para ICS asociada a la CVC. El objetivo fue crear un website para mejorar el conocimiento, la actitud y la percepción de los enfermeros sobre las recomendaciones basadas en la evidencia sobre prevención y control de infección. La puntuación de los test fue decepcionante, con acierto por debajo del $50 \%$. La situación actual puede ser mejorada con la discusión de los problemas potenciales.

Estudio realizado en los Estados Unidos (11) buscó evaluar la eficacia de una intervención basada en la evidencia para prevenir ICS asociada a la CVC en una UCI por medio de una observación pre y post test y vigilancia de la incidencia de ICS asociada a CVC. La intervención se dio por conferencias, uso de pósteres y autoestudio. La incidencia disminuyó de 4,9 a 2,1. Una intervención educativa resultó en una significativa y sustentada reducción en la incidencia de ICS asociada a CVC.

Otro estudio realizado en los Estados Unidos ${ }^{(12)}$ en una $\mathrm{UCI}$, utilizó una intervención completando un paquete de medidas y uso de ilustraciones. La incidencia de ICS asociada a CVC pasó de 10.8 a 3.7, luego a 2.8 llegando a cero.La intervención educativa fue eficaz en la reducción de la ICS asociada a CVC y dio soporte para una cultura de tolerancia cero a la infección.

En otro estudio realizado en los Estados Unidos ${ }^{(13)}$ se buscó verificar si un programa educativo puede disminuir el número de ICS asociada a CVC en la UCI. Se observaron múltiples deficiencias en una auditoría a la cabecera del paciente después de la realización de un programa educativo. Tras una intervención a la cabecera del paciente con uso de figuras, charlas y demostración práctica abarcando la inserción y el mantenimiento del CVC se observó mejoría en la documentación, en la elección del lugar de inserción del catéter, en el curativo y en medidas de seguridad, mas no mejoró la higiene de las manos y el uso de máxima precaución de barrera estéril. Fue entonces cuando la incidencia de ICS asociada a CVC disminuyó de 3,4 a 2,7. La adherencia a las buenas prácticas permitió la disminución del número de casos y la frecuencia de ICS asociada a CVC.

Estudio realizado en los Estados Unidos ${ }^{(14)}$ buscó determinar si un programa de educación puede reducir la incidencia de ICS asociada a CVC. Se realizó una observación antes y después de la intervención. La incidencia al principio era de 9,4 y disminuyó a 5,5 y los costos se redujeron de 1.533 .000 a 1.036 .000 dólares. Una intervención enfocada a la educación de los profesionales que prestan cuidados para 
prevención de ICS asociada a CVC puede llevar a una reducción considerable en la incidencia de infección, en los costos derivados de cuidados médicos y en la morbidad relacionada con el uso de CVC.

Estudio realizado en Grecia ${ }^{(15)}$ con una población de 345 médicos y enfermeros que respondieron a un cuestionario para evaluar el conocimiento teórico sobre prácticas de prevención de ICS asociadas a CVC en una UCI. El porcentaje medio de aciertos en las tres partes del cuestionario fue $42,9 \% \pm 16,2 \%, 86,9 \% \pm 9,5 \%$ y $85,4 \% \pm 7,2 \%$. En las preguntas referentes a la realización del procedimiento respondidas por los médicos, $13,6 \%$ de ellos respondieron correctamente. Ser del sexo femenino y estar familiarizado con medidas para prevención de infecciones asociadas al CVC permitió mayor puntuación en la parte del cuestionario que evaluó la adhesión a las prácticas específicas en relación a la inserción; en cuanto al hecho de ser enfermero se asoció a mayor puntuación en la parte relacionada con los cuidados con el mantenimiento y evaluación del CVC. Los resultados sugieren que existe una mayor necesidad de conocimiento teórico y mejora de las prácticas relativas al cuidado con el CVC. Programas educativos pueden ayudar en esta mejora.

En un estudio realizado en Brasil ${ }^{(16)}$, en la ciudad de São Paulo, fueron analizados los procedimentos y la incidencia de ICS en 144 pacientes distribuidos en dos grupos, uno control y otro experimental. El objetivo fue analizar el impacto de la implantación de un protocolo de inserción, mantenimiento y retirada de CVC para prevención de ICS. Con la utilización del protocolo se obtuvo una disminución en 23,1 en la densidad de ICS mostrando el impacto del protocolo.

Estudio realizado en España ${ }^{(17)}$ a través del uso de cuestionario y observación para evaluar el conocimiento de las enfermeras de cuidados intensivos del sur de Europa sobre las directrices basadas en la evidencia para la prevención de PAVM y comparar estos resultados en una perspectiva paneuropea. La puntuación media global fue de $45,1 \%$. El conocimiento de las enfermeras de cuidados intensivos del sur de Europa se mostró pobre, pero significativamente mejor que en los países paneuropeos.

Un estudio realizado en los Estados Unidos ${ }^{(18)}$ buscó verificar si la construcción de un panel electrónico es capaz de mejorar la adherencia al paquete de medidas y reducir la incidencia de PAVM en una unidad intensiva quirúrgica. La adherencia al paquete de medidas fue analizada tras la construcción del panel y mejoró de 39\% a 89\%. La incidencia mejoró en $15,2 \%$. La construcción de un panel electrónico mejoró la adherencia al paquete de medidas y redujo la incidencia de PAVM.

Estudio realizado en Bélgica ${ }^{(19)}$ en el que se desarrolló un cuestionario para evaluar el conocimiento de las enfermeras de Unidad de Cuidados Intensivos (UCI) sobre directrices basadas en evidencia para prevención de PAVM. Con el análisis de las respuestas fue posible detectar equívocos generalizados entre las enfermeras. Los resultados pueden ser usados para la realización de programas educativos para prevención de PAVM.

Estudio realizado en Italia ${ }^{(20)}$ utilizó la aplicación de un cuestionario para evaluar el conocimiento de enfermeros y destacar las causas que dificultan la adherencia a las directrices para prevención de PAVM. Solo 22,6\% presentaron conocimiento y aplicación satisfactoria de estrategias para la prevención de PAVM, 54,8\% declararon que están mal informados, $80,9 \%$ dijeron que aplican una o más estrategias, 17,9\% relataron que no aplican ninguna estrategia. Las razones dadas para la no aplicación 
de las estrategias fueron: falta de protocolos $(31,5 \%)$, falta de recursos necesarios $(14,3 \%)$, no concordar con el método $(3,2)$, costos elevados $(2,6 \%)$, posibilidad de provocar efectos incómodos a los pacientes (1\%) y otros efectos $(0,6 \%)$. Las estrategias para prevención de PAVM son ampliamente aplicadas por los enfermeros, mas no de forma responsable e informada. Es importante asegurar que los enfermeros reciben formación continua y están involucrados en la elaboración y actualización de los protocolos.

Estudio realizado en los Estados Unidos ${ }^{(21)}$ durante un Seminario de Educación para Enfermeros buscó evaluar el conocimiento de las enfermeras de UCI sobre buenas prácticas dirigidas a pacientes adultos que reciben ventilación mecánica. $82 \%$ relataron conformidad con las orientaciones de lavado de manos, $75 \%$ relataron el uso de guantes, 1/2 relató elevar la cabecera de la cama, 1/3 relató realizar aspiración subglótica y $1 / 2$ trabajar con protocolo de cuidado oral. Las directrices para prevención de PAVM del CDC son ejecutadas con más consistencia en los hospitales que trabajan con la utilización de protocolos y la reducción de la incidencia de PAVM puede ser conseguida por la mayor ejecución de protocolos de cuidados orales.

Investigación realizada en los Estados Unidos ${ }^{(22)}$ con la aplicación de un test para evaluar el conocimiento de las enfermeras que actúan en $\mathrm{UCl}$ sobre el uso de paquete de cuidados para prevenir PAVM tras reunirse presentando una revisión de literatura sobre el asunto. Tras las sesiones educativas la actuación de las enfermeras mejoró en 8 de los 10 items. Sesiones de educación destinadas a informar a los enfermeros sobre el paquete de medidas para la prevención de PAVM tiene efecto significativo sobre la adquisición de conocimiento y mejora de la práctica.

Es posible ver con la presentación de los estudios, el enfoque reportado para estrategias como charlas, pósteres, paquetes de medidas, auditoría a la cabecera del enfermo, ilustraciones, figuras, demostraciones prácticas, protocolos, paneles electrónicos, website y evaluación del conocimiento de los profesionales esta última utilizada como forma de posibilitar al profesional el contacto con las directrices actualizadas sobre las recomendaciónes para la prevención de las infecciones hospitalarias y estimular la adquisición de nuevos conocimientos.

La busca de estrategias innovadoras evidencia una preocupación en romper con una forma tradicional de educación buscando nuevas formas de involucrar y estimular al profesional en la adherencia a las medidas que juntas o aisladamente influyen en la ocurrencia de las infecciones hospitalarias.

Los estudios muestran el impacto de estas estrategias en los índices de estas infecciones. Los estudios presentados abordan las intervenciones educativas por topografía de infección. Ocho artículos están relacionados con la topografía corriente sanguínea y cinco con el tracto respiratorio lo que muestra una sistematización y foco en determinado tipo de infección que presenta riesgos y características propias, inclusive en relación con las medidas preventivas, con posibilidad de abordaje de forma individual.

\section{Introducción de Nuevas Tecnologías en Salud}

Se presentan dos estudios sobre cómo las tecnologías en este caso, cateteres y profesionales pueden influir en la disminuvión de los casos de infección hospitalaria. 


\begin{tabular}{|c|c|c|c|}
\hline AUTORES & AÑO & $\begin{array}{l}\text { BASE DE DATOS/ } \\
\text { FUENTE DE }\end{array}$ & TÍTULO \\
\hline $\begin{array}{l}\text { Alonso-Echanove J } \\
\text { Edwards JR } \\
\text { Richards MJ } \\
\text { Brennan P } \\
\text { Venezia RA } \\
\text { Keen J }{ }^{(23)} \text { et al }\end{array}$ & 2003 & $\begin{array}{ll}\text { MEDLINE/ } & \text { Infect } \\
\text { Control } & \text { Hosp } \\
\text { Epidemiol } & \\
\end{array}$ & $\begin{array}{l}\text { Effect of nurse staffing } \\
\text { and antimicrobial- } \\
\text { impregnated central } \\
\text { venous catheters on risk } \\
\text { for bloodstream } \\
\text { infections in intensive } \\
\text { care units }\end{array}$ \\
\hline $\begin{array}{l}\text { Gutiérrez-Zufiaurre } \\
\text { MN } \\
\text { García-Rodríguez JA } \\
(24)\end{array}$ & 2006 & $\begin{array}{l}\text { IBECS/ Rev Esp } \\
\text { Quimioter }\end{array}$ & $\begin{array}{lr}\text { Encuesta multicénctrica } \\
\text { nacional sobre utilización } \\
\text { de } & \text { antibióticos } \\
\text { intravenosos } & \end{array}$ \\
\hline
\end{tabular}

Cuadro III - Distribución de las bibliografías de la categoría temática "Introdución de Nuevas Tecnologías en Salud".

Estudio realizado en los Estados Unidos ${ }^{(23)}$ en el que se observa que el catéter impregnado con antimicrobiano disminuyó el riesgo para ICS asociada a CVC entre los pacientes en los que este catéter fue utilizado para administrar nutrición parenteral- NPT. Los factores de riesgo para ICS asociada a CVC fueron uso de NPT en catéter no impregnado con antimicrobiano, pacientes cuidados por enfermeras que realizaban rotación en $60 \%$ del tiempo de uso del CVC, pacientes que no usaron antibiótico dentro de las primeras 48 horas tras la inserción del catéter y pacientes que no despertaron por $70 \%$ o más en la duración del catéter. Catéter Venoso Central de Inserción Periférica (PIIC) fue asociado a menor riesgo de ICS asociada a CVC. Utilizar catéter impregnado con antimicrobiano en pacientes en uso de NPT, limitar la utilización de rotación entre enfermeras y la utilización de PICC puede reducir la ICS asociada a CVC.

En estudio realizado en España ${ }^{(24)}$ se recogieron datos sobre el manejo del catéter central y periférico y sobre la administración de antibióticos en hospitales españoles. Los resultados indican que el mantenimiento, inserción, eliminación y administración de antibióticos son realizados por el personal da enfermería siguiendo protocolos establecidos principalmente sobre el cuidado con el catéter. La utilización de antibiótico intravenoso es una parte importante de la asistencia y de los gastos en salud.

Cuando se ponen en práctica todas las recomendaciones para la prevención de infecciones, la utilización de nuevas tecnologías, el catéter impregnado con antimicrobiado y el uso de PICC, se implementan beneficiosamente en la asistencia y así se destaca en los artículos.

\section{Higiene Oral}

Se describen seis artículos que relacionan la higiene oral con la reducción de infecciones hospitalarias, las formas de realización de la técnica que causan más impacto y la preocupación con los riesgos derivados de esta acción. 


\begin{tabular}{|c|c|c|c|}
\hline AUTORES & AÑO & $\begin{array}{lr}\text { BASE DE DATOS/ } \\
\text { FUENTE } \\
\text { DATOS }\end{array}$ & TÍTULO \\
\hline Ames $\mathrm{NJ}^{(25)}$ & 2011 & $\begin{array}{l}\text { MEDLINE/ Am J } \\
\text { Crit Care }\end{array}$ & $\begin{array}{l}\text { Evidence to support } \\
\text { brushing in critically ill } \\
\text { patients }\end{array}$ \\
\hline $\begin{array}{l}\text { Feider LL } \\
\text { Mitchell P } \\
\text { Bridges } \mathrm{E}^{(26)}\end{array}$ & 2010 & $\begin{array}{l}\text { MEDLINE/ Am J } \\
\text { Crit Care }\end{array}$ & $\begin{array}{l}\text { Oral care practices for } \\
\text { orally intubated critically } \\
\text { ill adults }\end{array}$ \\
\hline $\begin{array}{l}\text { Munro CL } \\
\text { Grap MJ } \\
\text { Jones DJ } \\
\text { McClish DK } \\
\text { Sessler CN }\end{array}$ & 2009 & $\begin{array}{l}\text { MEDLINE/ Am J } \\
\text { Crit Care }\end{array}$ & $\begin{array}{l}\text { Chlorhexidine, } \\
\text { toothbrushing and } \\
\text { preventing ventilator- } \\
\text { associated pneumonia in } \\
\text { critically ill adults }\end{array}$ \\
\hline $\begin{array}{l}\text { Berry AM } \\
\text { Davidson PM } \\
\text { Masters J } \\
\text { Rolls K }\end{array}$ & 2007 & $\begin{array}{l}\text { MEDLINE/ Am J } \\
\text { Crit Care }\end{array}$ & $\begin{array}{l}\text { Systematic literature } \\
\text { review of oral hygiene } \\
\text { practices for intensive } \\
\text { care patients receiving } \\
\text { mechanical ventilation }\end{array}$ \\
\hline $\begin{array}{l}\text { Grap MJ } \\
\text { Munro CL } \\
\text { Ashtiani B } \\
\text { Bryant S }\end{array}$ & 2003 & $\begin{array}{l}\text { MEDLINE/ Am J } \\
\text { Crit Care }\end{array}$ & $\begin{array}{l}\text { Oral care interventions in } \\
\text { critical care: frequency } \\
\text { and documentation }\end{array}$ \\
\hline $\begin{array}{l}\text { Kjonegaard R } \\
\text { Fields W } \\
\text { King ML }{ }^{(30)}\end{array}$ & 2009 & $\begin{array}{l}\text { MEDLINE/ Am J } \\
\text { Crit Care }\end{array}$ & $\begin{array}{l}\text { Current practice in airway } \\
\text { management: } \\
\text { descriptive evaluation }\end{array}$ \\
\hline $\begin{array}{l}\text { Jones DJ } \\
\text { Munro CL }\end{array}$ & 2008 & $\begin{array}{l}\text { MEDLINE/ } \\
\text { Intensive Crit Care } \\
\text { Nurs }\end{array}$ & $\begin{array}{l}\text { Oral care and risk of } \\
\text { bloodstream infections in } \\
\text { mechanically ventilated } \\
\text { adults: a reviw }\end{array}$ \\
\hline $\begin{array}{l}\text { Jones DJ } \\
\text { Munro CL } \\
\text { Grap MJ } \\
\text { Kitten T } \\
\text { Edmond M }{ }^{(32)}\end{array}$ & 2010 & $\begin{array}{ll}\text { MEDLINE/ Heart } \\
\text { Lung }\end{array}$ & $\begin{array}{l}\text { Oral care and bacteremia } \\
\text { risk in mechanically } \\
\text { ventilated adults }\end{array}$ \\
\hline
\end{tabular}

Cuadro IV - Distribución de las bibliografias de la categoría temática "Higiene Oral".

Estudio realizado en los Estados Unidos ${ }^{(25)}$ utilizando la revisión de literatura en banco de datos para resumir la evidencia que da soporte a la eficacia del cepillado de dientes en adultos y niños en estado crítico que recibieron ventilación mecánica. Las posibles razones de la falta de beneficio del cepillado dental mostrada por los ensayos clínicos son discutibles. Se sugirieron recomendaciones para nuevos estudios que den mayor énfasis a la evidencia que da soporte a esta intervención.

Estudio realizado en los Estados Unidos ${ }^{(26)}$ con 347 miembros de la Asociación Americana de Cuidados Intensivos (AACl) y que trabajan en UCl buscó describir las prácticas de higiene oral realizadas por estos enfermeros en los pacientes intubados y enfermos críticos y comparar estas prácticas con las recomendaciones del manual de procedimientos de la AACN y el Centro de Control de Enfermedades (CDC). El cuidado oral es realizado cada 2 o 4 horas generalmente con torundas de espuma. La higiene oral fue considerada como una prioridad. Enfermeros con más años de experiencia en cuidados intensivos realizan cuidados bucales con mayor frecuencia. 
Enfermeros graduados aspiran la boca antes del tubo endotraqueal y tras la higiene oral con más frecuencia de lo que lo hacemos otros enfermeros. Enfermeros que trabajaban utilizando protocolos relataron e la conducta indicada para la utilización de cepillo (63\%), pasta de dientes (40\%), uso de torunda de espuma (90\%), solución oral de clorexidina (49\%), y en cuanto al momento de la realización del cepillado, (84\%) en el momento de la aspiración y (73\%) en el momento de la evaluación de la cavidad oral. Prácticas y conductas difieren en todos los items. Existen discrepancias entre las prácticas relatadas. La gestión del cuidado parece estar presente, mas no es utilizada de manera adecuada.

Estudio realizado en los Estados Unidos ${ }^{(27)}$ dividió 547 pacientes en cuatro grupos para los siguientes tratamientos: torunda de clorexidina solución oral dos veces al día, cepillado 3 veces al día, cepillado y clorexidina, y grupo con tratamiento usual. En tres días de análisis, 249 pacientes permanecieron en el estudio. Cuando los datos sobre todos los pacientes fueron analizados juntos, indicaron que la clorexidina redujo significativamente la incidencia de PAVM a partir del tercer día.

Estudio realizado en Australia ${ }^{(28)}$ utilizó la revisión de literatura para evaluar la mejor evidencia disponible para realizar higiene oral en pacientes de cuidados intensivos que son sometidos a ventilación mecánica. La búsqueda arrojó 55 artículos. A pesar de ser la temática de extrema importancia, el nivel de evidencia de los estudios controlados y aleatorios y las revisiones sistemáticas de alta calidad que podrían fundamentar la práctica clínica son escasas.

Estudio realizado en los Estados Unidos ${ }^{(29)}$ con utilización de formulario para identificar frecuencia y documentación de intervenciones para higiene oral en UCI. La mayoría de los enfermeros respondieron que realizaban higiene oral 2 o 3 veces en los pacientes no intubados y 5 veces o más en los pacientes intubados. Sin embargo, en el análisis de las historias clínicas se verificó anotación de higiene oral en una media de 1,6 veces por paciente en 24 horas. El uso de pasta y cepillo de dientes fue mayor en pacientes no intubados y el uso de esponja para limpieza de los dientes fue mayor en pacientes intubados. En una escala de cero a cien la higiene oral fue señalada como prioridad en una media de 5,3 puntos. A pesar de las evidencias de que las esponjas son ineficaces para la eliminación de placas dentales, las esponjas permanecen como principal herramienta de higiene oral especialmente para pacientes intubados en UCI. Los enfermeros relatan que hacen higiene oral con frecuencia, mas pocas de las intervenciones son relatadas. Intervenciones educativas son necesarias para garantizar una adecuada realización de higiene oral y estudios de enfermería para delinear el mejor procedimiento a ser utilizado para todos los pacientes.

Estudio desarrollado en los Estados Unidos ${ }^{(30)}$ buscó determinar la práctica actual y las diferencias de conducta entre enfermeros y fisioterapeutas en el tratamiento de pacientes en ventilación mecánica. El estudio describió y comparó las técnicas de aspiración y los cuidados con vía aérea. Enfermeros utilizan para hiperoxigenación el porpio ventilador mientras fisioterapeutas usan ambu. Los fisioterapeutas instilan solución fisiológica para lavar el sistema cerrado de aspiración con más frecuencia que los enfermeros. Los enfermeros aspiran la secreción de la nariz y de la boca, lavan los dientes de los pacientes con cepillo y utilizan hisopo para limpiar la boca más frecuentemente que los fisioterapeutas. Existe diferencia entre los cuidados prestados por enfermeros y fisioterapeutas para prevención de PAVM. 
Estudio realizado en los Estados Unidos ${ }^{(31)}$ con utilización de revisión de literatura buscó la relación entre la manipulación de la cavidad oral y el desarrollo de bacteriemia en adultos mecánicamente ventilados. Nueve artículos cumplieron los criterios de inclusión y fueron analizados. No fue posible establecer el origen de bacteriemia en la mayoría de los estudios a pesar de ser posible conocer los gérmenes que causaron la infección. Son necesarias investigaciones adicionales para entender la relación entre prácticas de higiene oral y bacteriemia en adultos mecánicamente ventilados.

Estudio realizado en los Estados Unidos ${ }^{(32)}$ se dio por un análisis microbiológico para identificar el tipo del DNA de microorganismos a partir de cultivos orales y de sangre colectados inmediatamente antes y un minuto y treinta segundos tras el cepillado. Se buscó así evaluar la incidencia y el significado de la bacteriemia transitoria derivada del cepillado dental en adultos mecánicamente ventilados. Ninguno de los individuos tenía evidencia de bacteriemia transitoria. La intervención de cepillado dental no indujo bacteriemia transitoria en esta población de pacientes.

La higiene oral es recomendada por las directrices nacionales e internacionales como acción para interferir en la ocurrencia de PAVM. Los artículos evidencian la preocupación con la mejor forma de realización de la técnica, las posibilidades de utilización de los materiales, las dificultades presentadas por los profesionales en la adherencia a este cuidado y los riesgos potenciales.

\section{Aspiración de la Secreción Gástrica}

El estudio seleccionado se relaciona con la aspiración de la secreción gástrica como importante acción para prevenir la PAVM.

\begin{tabular}{|c|c|c|c|}
\hline AUTORES & AÑO & $\begin{array}{l}\text { BASE DE DATOS/ } \\
\text { FUENTE } \\
\text { DATOS }\end{array}$ & TÍTULO \\
\hline $\begin{array}{l}\text { Metheny NA } \\
\text { Clouse RE } \\
\text { Chang YH } \\
\text { Stewart BJ } \\
\text { Oliver DA } \\
\text { Kollef MH }{ }^{(33)}\end{array}$ & 2006 & $\begin{array}{l}\text { MEDLINE/ Crit } \\
\text { Care Med }\end{array}$ & $\begin{array}{l}\text { Tracheobronchial } \\
\text { aspiration of gastric } \\
\text { contents in critically ill } \\
\text { tube-fed patients: } \\
\text { frequency, outcomes and } \\
\text { risk factors }\end{array}$ \\
\hline
\end{tabular}

Cuadro V - Distribución de las bibliografías de la categoría temática "Aspiración de la Secreción Gástrica".

Estudio realizado en los Estados Unidos ${ }^{(33)}$ durante dos años utilizando colecta de casi 6.000 secreciones traqueales durante el sondaje de rutina y pruebas de pepsina con el objetivo de describir la frecuencia de pepsina-positivos en secreción traqueal, los resultados de pneumonía clínica, el uso de recursos hospitalarios y factores de riesgo asociados con aspiración y pneumonía en una población de pacientes en estado crítico alimentados por catéter. De las muestras de secreción colectadas, $31,3 \%$ fueron positivas.

Por lo menos un evento de aspiración fue identificado en $88,9 \%$ de los pacientes $(n=320)$. La incidencia de pneumonía aumentó de $24 \%$ el primer día a $48 \%$ el cuarto día. Pacientes con pneumonía en el cuarto día tuvieon un porcentaje mayor de pepsina-positiva en la secrección traqueal que los sin diagnóstico de pneumonía. Para 
los pacientes con pneumonía el tiempo en la UCl y el tiempo de soporte ventilatorio fueron mayores.

La cabecera baja de la cama era un factor de riesgo para la aspiración, para vómitos, alimentación gástrica, Escala de Coma de Glasgow < que 9 y enfermedad de reflujo gastroesofágico. Los factores de riesgo independientes fueron broncoaspiración, uso de agentes paralizantes y altos niveles de sedación. La aspiración del contenido gástrico es común en pacientes en estado crítico alimentados por catéter, es un importante factor de riesgo para pneumonía y, además, lleva al aumento de los costos hospitalarios.

\section{Cambio de Decúbito}

El estudio seleccionado presenta el cambio de decúbito como importante acción en la prevención de la PAVM.

\begin{tabular}{|c|c|c|c|}
\hline AUTORES & AÑO & $\begin{array}{l}\text { BASE DE DATOS/ } \\
\text { FUENTE } \\
\text { DATOS }\end{array}$ & TÍTULO \\
\hline $\begin{array}{l}\text { Goldhil DR } \\
\text { Imhoff M } \\
\text { McLean B } \\
\text { Waldmann C }\end{array}$ & 2007 & $\begin{array}{l}\text { MEDLINE/ Am } \mathrm{J} \\
\text { Crit Care }\end{array}$ & $\begin{array}{l}\text { Rotational bed therapy to } \\
\text { prevent and treat } \\
\text { respiratory complications: } \\
\text { a review and meta- } \\
\text { analysis }\end{array}$ \\
\hline
\end{tabular}

Cuadro IV - Distribución de las bibliografias de la categoría temática "Cambio de Decúbito".

Meta-análisis realizado en Gran Bretaña ${ }^{(34)}$ buscó revisar el efecto del cambio de decúbito en la prevención y/o tratamiento de complicaciones respiratorias en pacientes enfermos críticos. De acuerdo con los estudios lo que normalmente se hacía era cambio de decúbito cada dos horas sugiriendo que este tipo de movilización disminuía la incidencia de pneumonía y puede ser útil para impedir complicaciones respiratorias.

Presentadas las categorías 5 y 6 para discusión es posible entender que evidenciar la importancia de una acción de enfermería para la prevención de infecciones hospitalarias es de fundamental importancia para el entendimiento del profesional en relación a su práctica y un incentivo a la adhesión a la realización de estas acciones de forma consciente y responsable.

\section{Elevación de la Cabecera de la cama}

Presentación de dos artículos sobre el efecto de la elevación de la cabecera de la cama y la precisión del enfermero para la realización de esta acción.

\begin{tabular}{|c|c|c|c|}
\hline AUTORES & AÑO & $\begin{array}{lr}\text { BASE DE DATOS/ } \\
\text { FUENTE } \\
\text { DATOS }\end{array}$ & TÍTULO \\
\hline $\begin{array}{l}\text { Grap MJ } \\
\text { Munro CL } \\
\text { Hummel RS }\end{array}$ & 2005 & $\begin{array}{l}\text { MEDLINE/ Am } \mathrm{J} \\
\text { Crit Care }\end{array}$ & \begin{tabular}{lrr} 
Effect of & \multicolumn{2}{r}{ backrest } \\
elevation & on & the \\
development & & of
\end{tabular} \\
\hline
\end{tabular}




\begin{tabular}{l|l|l|l}
\hline $\begin{array}{l}\text { Elswick RK Jr } \\
\text { McKinney JL } \\
\text { Sessler CN }{ }^{(35)}\end{array}$ & & & $\begin{array}{l}\text { ventilator-associated } \\
\text { pneumonia }\end{array}$ \\
\hline $\begin{array}{l}\text { Dillon A } \\
\text { Munro CL } \\
\text { Grap MJ (36) }\end{array}$ & 2002 & $\begin{array}{l}\text { MEDLINE/ Am J J } \\
\text { Crit Care }\end{array}$ & $\begin{array}{l}\text { Nurses' accuracy in } \\
\text { estimating backrest } \\
\text { elevation }\end{array}$ \\
\hline
\end{tabular}

Cuadro IV - Distribución de las bibliografías de la categoría temática "Elevación de la Cabecera de la cama".

Estudio realizado en los Estados Unidos ${ }^{(35)}$ buscó describir la relación entre la elevación de la cabecera de la cama y el desarrollo de PAVM. Para esto se determinó la incidencia de PAVM y medida de la cabecera de la cama continuamente con un sistema de transductor. La media de elevación de la cabecera de la cama para el período de estudio fue de 21,7 grados. Con menos de 30 grados en $72 \%$ del tiempo y con menos de 10 grados en $39 \%$ del tiempo. La media de infección clínica pulmonar aumentó, mas no significativamente, y la elevación de la cabecera de la cama no tuvoe efecto directo sobre la incidencia de PAVM. Los pacientes pasaron la mayor parte del tiempo con la cabecera de la cama a menos de 30 grados. Solamente la combinación de la cabecera baja y la gravedad de la enfermedad afectó el índice de PAVM.

Estudio realizado en los Estados Unidos ${ }^{(36)}$ con enfermeros que frecuentaban la Asociación Americana de Enfermeros de Cuidados Intensivos buscó determinar la diferencia entre ángulos de la cama estimados por los enfermeros, ángulos medidos en la cama y describir la relación entre características de los enfermeros y la precisión de sus estimaciones. Los enfermeros fueron precisos en estimar los ángulos de la cama. Otros estudios son necesarios para entender por qué las recomendaciones para elevación de la cama no son usadas en la práctica.

La acción enfocada en los artículos influye en la prevención de la PAVM cuando el paciente se encuentra en estado grave lo que es determinante para que esta acción esté inserta en un sector de enfermedades con enfermos en estado crítico. Es interesante percibir la habilidad de los profesionales de enfermería en la realización de técnicas pertinentes a su profesión y es importante concluir la existencia de cuestiones de la práctica que necesitan ser estudiadas.

\section{CONCLUSIÓN}

Con la realización de esta investigación, se concluye que las acciones y/o intervenciones de enfermería para prevención de infecciones hospitalarias en pacientes gravemente enfermos, que más aparecen en la literatura, son: la higiene de las manos, intervenciones educativas con énfasis en evaluación de conocimiento, charlas, demostraciones prácticas, uso de ilustraciones, paquetes de medidas, protocolos, paneles electrónicos, website, introducción de nuevas tecnologías en salud, higiene oral, aspiración de secreción gástrica, cambio de decúbito y elevación de la cabecera de la cama y ejercen influencia en la redución de la incidencia de infecciones hospitalarias.

En relación a las topografías, son siempre estudiadas separadamente, y se da mayor enfoque a la ICS asociada a CVC y a PAVM ya que tiene gran impacto en la morbidad y mortalidad de los pacientes en estado crítico. Se observó que la mayor parte de los estudios se realizaron en países extranjeros, principalmente en los Estados Unidos, lo 
que muestra la apremiante necesidad del desarrollo de estudios brasileños relativos a la temática.

\section{REFERENCIAS}

1. Fernandes AT. Infecção hospitalar e suas interfaces na área de saúde. São Paulo: Atheneu; 2000.

2. Agência Nacional de Vigilância Sanitária (ANVISA). Gerência Geral de Tecnologia em Serviço de Saúde. Gerência de Investigação e Prevenção de Infecções e Eventos Adversos. Indicadores nacionais de infecções relacionadas à assistência à saúde. Brasília; 2010. [acesso online em 2012 maio. 5]. Disponível em : www.anvisa.gov.br.

3. Gerência Geral de Tecnologia em Serviço de Saúde. Gerência de Investigação e Prevenção de Infecções e Eventos Adversos. Trato respiratório: critérios nacionais de infecções relacionadas à assistência à saúde. Brasília; 2009. [acesso online em 2012 maio. 5]. Disponível em : www.anvisa.gov.br.

4. Gerência Geral de Tecnologia em Serviço de Saúde. Gerência de Investigação e Prevenção de Infecções e Eventos Adversos. Corrente sanguínea: critérios nacionais de infecções relacionadas à assistência à saúde. Brasília; 2009. [acesso online em 2012 maio. 5]. Disponível em : www.anvisa.gov.br.

5. Waldow VR, Lopes MJM, Meyer, DE. Maneiras de cuidar, maneiras de ensinar: a enfermagem entre a escola e a prática profissional. Porto Alegre: Artes Médicas; 1995.

6. Souza MT, Silva MD, Carvalho R. Revisão integrativa: o que é e como fazer. Einstein. 2010; 8:102-6.

7. Silvino ZR. Quadro para coleta de dados de pesquisa bibliográfica em base de dados. 2012.

8. Hugonnet S, Perneger TV, Pittet D. Al. Alcohol-based handrub improves compliance with hand in intensive care units. Arch Intern Med.[Internet] 2002; May 13; 162 (9): 1037-43. Disponivel em: http://archinte.jamanetwork.com/article.aspx?articleid=211416.

9. Qushmaq IA, Heels-Ansdell D, Cook DJ, Loeb MB, Meade MO. Hand hygiene in intensive care unit: prospective observations if clinical practice. Pol Arch Med. [Internet] 2008; $118 \quad$ (10): 543-7. Disponivel em: http://www.ncbi.nlm.nih.gov/pubmed/19112814.

10. Vandijck DM, Labeau SO, Vogelaers DP, Blot SI. Prevention of nosocomial infections in intensive care patients. Nurs Crit Care. [Internet] 2010; Set-Out; 15 (5): 251-6. Disponivel em: http://www.ncbi.nlm.nih.gov/pubmed/20712670.

11. Warren DK, Zack JE, Cox MJ, Cohen MM, Fraser VJ. An educational intervention to prevent catheter-associated bloodstream infections in a nonteaching, community medical center. Crit Care Med. [Internet] 2003; Jul; 31 (7): 1959-63. Disponivel em: http://www.ncbi.nlm.nih.gov/pubmed/12847389.

12. Zack JE. Zeroing in on zero tolerance for central line-associated bacteremia. Am J Infect Control. [Internet] 2008; Dec; 36 (10): S176.e 1-2. Disponivel em: http://www.ncbi.nlm.nih.gov/pubmed/19084157.

13. Coopersmith CM, Zack JE, Ward MR, Sona CS, Scallon ME, Everett SJ. The impact if bedside behavior on catheter-related bacteremia in the intensive care unit. Arch Surg. [Internet] 2004; Fev; 139 (2): 131-6. Disponivel em: www.ncbi.nlm.nih.gov/pubmed/14769568.

14. Warren DK, Zack JE, Mayfield JL, Chen A, Prantice D, Fraser JV, Kollef MH. The effect of an education program on the incidence of central venous catheter-associated bloodstream infection in a medical ICU. Chest. [Internet] 2004; Nov; 126 (5): 1612-8. Disponivel em: www.ncbi.nlm.nih.gov/pubmed/15539735. 
15. Koutzavekiaris I, Vouloumanou EK, Gourni M, Rafailidis PI, Michalopoulos A, Falagas ME. Knowledge and practices regarding prevention of infections associated with central venous catheters: a survey of intensive care unit medical and nursing staff. Am J Infect Control. [Internet] 2011; Set; 39 (7): 542-7. Disponivel em: www.ncbi.nlm.nih.gov/pubmed/21496955.

16. Castilho LD, Medeiros MM, Carrasco HVCJ, Marvulo MML. Infecções de corrente sanguínea relacionadas ao cateter venoso central em terapia intensiva: ensaio clínico randomizado aberto. Nursing. [Internet] 2009; Set; 12 (136): 429-434. Disponivel em: www.nursing.com.br/paper.php? $\mathrm{p}=472$.

17. Llauradó M, Labeau S, Vandijck D, Rello J, Rosa A, Riera A et al. Southern european intensive care nurses' knowledge of evidence-based gudelines for preventing ventilator-associated pneumonia. Med Intensiva. [Internet] 2011; Jan-Fev; 35 (1): 6-12. Disponivel em: www.ncbi.nlm.nih.gov/pubmed/21122950.

18. Zaydfudim V, Dossett LA, Starmer JM, Arbogast PG, Feurer ID, Ray WA et al. Implementation of a real-time compliance dashboard to help reduce SICU ventilatorassociated pneumonia with the ventilator bundle. Arch Surg. [Internet] 2009; Jul; 144 (7): 656-62. Disponivel em: http://apicwv.org/docs/40.pdf.

19. Cason CL, Tyner T, Saunders S, Broome L. Nurses' implementation of guidelines for ventilator-associated pneumonia from the Centers for Disease Control and Prevention. Am J Crit Care. [Internet] 2007; Jan; 16 (1): 28-36. Disponivel em: www.ncbi.nlm.nih.gov/pubmed/17192524.

20. Biancofire G, Barsotti E, Catalani V, Landi A, Bind L, Urbani L et al. Nurses' knowledge and application of evidence-based guidelines for preventing ventilatorassociated pneumonia. Minerva Anestesiol. 2007; Mar; 73 (3): 129-34. Disponivel em: www.ncbi.nlm.nih.gov/pubmed/17115016.

21. Labeau S, Vandijck DM, Claes B, Van Aken P, Blot SI. Critical care nurse's knowledge of evidence-based guidelines for preventing ventilator-associated pneumonia: an evaluation questionnaire. Am J Crit Care. [Internet] 2007; Jul; 16 (4): 371-7. Disponivel em: www.ncbi.nlm.nih.gov/pubmed/17595369.

22. Tolentino-DelosReyes AF, Ruppert SD, Shiao SY. Evidence-based practice: use of the ventilator bundle to prevent ventilator-associated pneumonia. Am J Crit Care. 2007; Jan; 16 (1): 20-7. Disponivel em: www.ncbi.nlm.nih.gov/pubmed/17192523.

23. Alonso-Echanove J, Edwards JR, Richards MJ, Brennan P, Venezia RA, Keen J et al. Effect of nurse staffing and antimicrobial-impregnated central venous catheters on risk for bloodstream infections in intensive care units. Infect Control Hosp Epidemiol. [Internet] 2003; Dec; $24 \quad$ (12): 916-25. Disponivel em: www.ncbi.nlm.nih.gov/pubmed/14700407.

24. Gutiérrez-Zufiaurre MN, García-Rodríguez JÁ. Encuesta multicénctrica nacional sobre utilización de antibióticos intravenosos. Rev Esp Quimioter. [Internet] 2006; 19 (4): 349-356. Disponivel em: http://www.seq.es/seq/0214-3429/19/4/gutierrez z.pdf.

25. Ames NJ. Evidence to support brushing in critically ill patients. Am J Crit Care. [Internet] 2011; May; 20 (3): 242-50. Disponivel em: www.ncbi.nlm.nih.gov/pubmed/21532045.

26. Feider $L L$, Mitchell $P$, Bridges $E$. Oral care practices for orally intubated critically ill adults. Am J Crit Care. [Internet] 2010; Mar; 19 (2): 175-83. Disponivel em: www.ncbi.nlm.nih.gov/pubmed/20194614.

27. Munro CL, Grap MJ, Jones DJ, McClish DK, Sessler CN. Chlorhexidine, toothbrushing and preventing ventilator-associated pneumonia in critically ill adults. Am J Crit Care. [Intrnet] 2009; Sep; 18 (5):428-37. Disponivel em: www.ncbi.nlm.nih.gov/pubmed/19723863.

28. Berry AM, Davidson PM, Masters J, Rolls K. Systematic literature review of oral hygiene practices for intensive care patients receiving mechanical ventilation. Am $J$ 
Crit Care. [Internet] 2007; Nov; 16 (6): 552-62. Disponivel em: www.ncbi.nlm.nih.gov/pubmed/17962500.

29. Grap MJ, Munro CL, Ashtiani B, Bryant S. Oral care interventions in critical care: frequency and documentation. Am J Crit Care. [Internet] 2003; Mar; 12 (2): 113-8. Disponivel em: www.ncbi.nlm.nih.gov/pubmed/12625169.

30. Kjonegaard R, Fields W, King ML. Current practice in airway management: a descriptive evaluation. Am J Crit Care. [Internet] 2009; Mar; 19 (2): 168-73. Disponivel em: www.ncbi.nlm.nih.gov/pubmed/19383762.

31. Jones DJ, Munro CL. Oral care and risk of bloodstream infections in mechanically ventilated adults: a reviw. Intensive Crit Care Nurs. [Internet] 2008; Jun; 24 (3): 15261. Disponivel em: www.ncbi.nlm.nih.gov/pubmed/18403205.

32. Jones DJ, Munro CL, Grap MJ, Kitten T, Edmond M. Oral care and bacteremia risk in mechanically ventilated adults. Heart Lung. [Internet] 2010; Nov-Dec; 39 (6): S5765. Disponivel em: www.ncbi.nlm.nih.gov/pubmed/20598375.

33. Metheny NA, Clouse RE, Chang $\mathrm{YH}$, Stewart BJ, Oliver DA, Kollef $\mathrm{MH}$. Tracheobronchial aspiration of gastric contents in critically ill tube-fed patients: frequency, outcomes and risk factors. Crit Care Med. [Internet] 2006; Apr; 34 (4): 1007-15. Disponivel em: www.ncbi.nlm.nih.gov/pubmed/16484901.

34. Goldhil DR, Imhoff M, McLean B, Waldmann C. Rotational bed therapy to prevent and treat respiratory complications: a review and meta-analysis. Am $\mathrm{J}$ Crit Care. [Internet] 2007; Jan; 16 (1): 50-61. Disponivel em: www.ncbi.nlm.nih.gov/pubmed/17192526.

35. Grap MJ, Munro CL, Hummel RS, Elswick RK Jr, McKinney JL, Sessler CN. Effect of backrest elevation on the development of ventilator-associated pneumonia. Am J Crit Care. [Internet] 2005; Jul; 14 (4): 1007-15. Disponivel em: www.ncbi.nlm.nih.gov/pubmed/15980424.

36. Dillon A, Munro CL, Grap MJ. Nurses' accuracy in estimating backrest elevation. Am J Crit Care. [Internet] 2002; Jan; 11 (1): 34-7. Disponivel em: www.ncbi.nlm.nih.gov/pubmed/11785555.

ISSN 1695-6141 